\title{
Effect of mixing, concentration and temperature on formation of mesostructured solutions and their role in nucleation of DL-valine crystals
}

\author{
Anna Jawor-Baczynska ${ }^{a}$, Barry D. Moore ${ }^{b}$ and Jan Sefcik ${ }^{a}$ \\ ${ }_{5}$ Received (in $\left.X X X, X X X\right) X$ th $X X X X X X X X X 20 X X$, Accepted Xth $X X X X X X X X X 20 X X$ \\ DOI: 10.1039/b000000x
}

We report investigations on formation of mesostructured solutions in DL-valine/water/2-propanol mixtures and crystallization of DL-valine from these solutions. Mesostructured liquid phases, similar to those previously observed in aqueous solutions of glycine and DL-alanine, were observed using Dynamic

${ }_{10}$ Light Scattering and Brownian microscopy in both undersaturated and supersaturated solutions below a certain transition temperature. Careful experimentation was used to demonstrate that the optically clear mesostructured liquid phase comprising colloidal mesoscale clusters dispersed within bulk solution is thermodynamically stable and present in equilibrium with the solid phase at saturation conditions.

Solutions prepared by slow cooling contained mesoscale clusters with a narrow size distribution and

15 mean hydrodynamic diameter around $200 \mathrm{~nm}$. Solutions of identical compositions prepared by rapid isothermal mixing of valine aqueous solutions with 2-propanol contained mesoscale clusters which were significantly larger than those observed in slowly cooled solutions. The presence of larger mesoscale clusters was found to correspond to faster nucleation. Observed induction times were strongly dependent on the rapid initial mixing step, although solutions were left undisturbed afterwards and induction times

20 observed were up to two orders of magnitude longer than the initial mixing period. We propose that mesoscale clusters above a certain critical size are likely locations of productive nucleation events.

\section{Introduction}

Crystallization is widely used for particle formation, purification and separation in the chemical and pharmaceutical industries. ${ }_{25}$ Phase separation of a solute from supersaturated solution to form a crystalline phase involves two main steps: nucleation, the birth of a stable crystal nucleus, and crystal growth, the addition of material onto an existing crystal lattice. In the last twenty years significant effort has been made to better understand the 30 mechanism of the nucleation process and a wide variety of techniques has been used to probe the relationship between molecular self-organization in solutions and melts, formation of nuclei and the resultant crystal polymorphs. Molecular selforganization due to solute-solute and solute-solvent interactions

35 have been examined in undersaturated, saturated and supersaturated crystallizing solutions using X-ray Small-Angle Scattering (SAXS) ${ }^{1-3}$, Wide-Angle X-ray Diffraction (WAXD) ${ }^{1}$, Fourier Transform Infrared Spectroscopy (FTIR) ${ }^{4}$, Nuclear Magnetic Resonance (NMR) ${ }^{5}$, Small Angle Neutron Scattering ${ }_{40}(\mathrm{SANS})^{6}$, Empirical Potential Structure Refinement (EPSR) ${ }^{7}$ and molecular modelling 8,9 . A common finding from these studies has been that solutes associate to form clusters in supersaturated solutions ${ }^{10-14}$. However, identification of a direct relationship between the solute molecular clusters and subsequent formed 45 crystal has been found to be challenging ${ }^{15-18}$. Two recently published reviews by Davey et al. and Gebauer at al. ${ }^{19,20}$ discuss various molecular self-assembly pathways in nucleating solutions including liquid-liquid macroscopic phase separation as well as formation the pre-nucleation clusters in a wide range of organic 50 and inorganic systems. A number of experimental and theoretical studies have pointed towards the formation of liquid-like clusters as the first step towards crystal nucleation ${ }^{21-25}$. Recent studies of crystal nucleation of various small molecules highlighted nonclassical mechanisms including formation of pre-nucleation 55 clusters or colloidal scale intermediates on the crystal nucleation pathway $^{20,26}$.

Despite numerous experimental and theoretical studies of nonclassical nucleation mechanisms of both small and large molecules many questions remain open regarding kinetics and 60 thermodynamics of formation of liquid-like mesospecies or prenucleation clusters and what are mechanisms of solid phase formation in such clusters. The nature of mesoscale clusters is not well understood and it is often not clear if they present a separate phase from surrounding solution or not, if they are in 65 equilibrium with surrounding solution or not, or if there is an energetic barrier to their formation or not. Better understanding the link between the pre-nuclation clusters, intermediate structures in solutions (under-and supersaturated) and molecular organisation in final crystalline product will provide crucial 70 insight required for better control of final particle attributes such 
as purity, morphology, polymorphism, size and size distribution. Metastable microphases with liquid-like properties have been observed in concentrated protein solutions ${ }^{11,21,23}$ but have also been reported in small molecule systems ${ }^{2}$. Macroscopic liquid5 liquid phase separation during a crystallization process has been also observed in certain classes of macromolecular systems such as proteins ${ }^{28-31}$ and polymers ${ }^{32,33}$. Bonnett et al. have studied the liquid-liquid phase separation during cooling induced crystallization of an organic substance from solvent mixtures ${ }^{27}$. 10 Formation of quasi-emulsions (consisting of apparently stable droplets with diameter of 100-1000 nm) during antisolvent precipitation of small organic compounds was reported by Wang et al. ${ }^{34}$ The optically clear quasi-emulsion can increase induction time, stabilizing crystallizing solution for days and can have 15 impact on polymorph formation ${ }^{35}$. Recent studies of effects of fluid shear on kinetics of primary nucleation pointed towards the possible role of colloidal scale clusters in crystal nucleation in butylparaben solutions in ethanol ${ }^{36}$ and glycine solutions in water $^{25}$

${ }_{20}$ Recently there have been a number of reports describing the formation of mesostructured crystals and their transformation to single crystals via non-classical pathways. Mesocrystals have been reported to be formed with both inorganic salts and organic compounds, usually but not exclusively in the presence of 25 stabilising polymers ${ }^{37-42}$. A system where mesocrystals were formed without polymers is that of the amino-acid DL-alanine in aqueous solution. Our previous study of undersaturated and supersaturated aqueous solution of DL-alanine revealed that mesoscale clusters with the size range from 100 to $300 \mathrm{~nm}$ exist 30 well below solid-liquid equilibrium concentration (saturation limit). It has been concluded that the mesoscale clusters are not a separate phase and the system has been described as a thermodynamically stable mesostructured liquid containing solute-rich domains dispersed within bulk solution ${ }^{43}$.

$35 \mathrm{We}$ have previously reported presence of submicron size mesospecies in supersaturated aqueous glycine solutions and their likely role in nucleation of glycine crystals ${ }^{44}$. We have also previously studied crystallization of DL-valine from 2-propanolwater solutions using Small Angle Static Light Scattering and 40 turbidimetry measurements, which indicated presence of mesoscale clusters in optically clear supersaturated solutions ${ }^{45}$. Here we further investigated crystallization of DL-valine from 2propanol-water solutions, mesoscale clusters in these solutions and their role in nucleation of DL-valine crystals, using either 45 slow cooling or rapid initial mixing of valine aqueous solutions with 2-propanol, followed by isothermal crystallization.

\section{Experimental}

Materials: All chemicals and solvents were of laboratory reagent grade and were used without further purification: 2-propanol 50 (VWR International Ltd.), DL-valine, puriss. $\geq 99.0 \%$, NT (Fluka). Deionised water was supplied from an in-house Millipore Water System.

Dynamic Light Scattering: The mean hydrodynamic diameter of 55 clusters was determined with an HPPS Malvern Instruments, HPP5001, equipped with temperature control, using photon correlation spectroscopy in backscattering mode, at a scattering angle of $173^{\circ}$ and laser light wavelength of $632 \mathrm{~nm}$. Autocorrelation function decays were fitted using a cumulant 60 method. The preparation of samples for DLS had to be conducted with the utmost care because scattering artefacts can be introduced while the species of interest can be inadvertently removed. It was particularly important to check the compatibility of the solvents and mixtures with all contacted materials and to 65 ensure filtration was carried out rigorously on all starting solutions and solvents but not on the crystallizing samples.

\section{Brownian microscopy/Nanoparticle Tracking Analysis:}

Cluster size distributions were determined with a Nanosight 70 LM10 instrument with temperature control unit. Nanosight NTA2.1 software was used to analyze videos and calculate the size and concentration of nanodroplets. The camera setting of the instrument was set using the 'Autosettings' option on the software to allow the software to optimize the shutter and gain 75 settings. The sample was introduced into the viewing unit, and an image of the particles' scattering of the laser light was captured by a CCD camera attached to a microscope. A video of the sample was recorded and processed, with each observed individual particle 'tracked' by the Nanoparticle Tracking 80 Analysis software. The diffusion coefficient from the mean squared displacement of the particle tracked was calculated, and substituted into the Stokes-Einstein equation to obtain the particle hydrodynamic diameter.

${ }_{85}$ Solubility measurements: The solubility of DL-valine in water-2propanol solvents mixture $(1: 1, \mathrm{v} / \mathrm{v})$ was determined in a temperature range from $25^{\circ} \mathrm{C}$ to $55^{\circ} \mathrm{C}$ using HPLC. Samples were prepared at fixed temperature in incubator by mixing an excess of crystalline DL-valine with solvent mixture (water-2-propanol, $901: 1, \mathrm{v} / \mathrm{v})$. The samples were left to equilibrate in the incubator whilst being continually stirred using the magnetic mixer plate for 24 hours at the set temperature being investigated. The supernatant solution was separated by filtration using a glass syringe and disposable $0.1 \mu \mathrm{m}$ PTFE syringe filters. $2 \mathrm{ml}$ of the 95 filtered sample was then added to another vial and diluted with $2 \mathrm{ml}$ of a pre-filtered 1:12-propanol/water mixture. The filtration step was also carried out in the incubator, all equipment used in this step had been left overnight in the incubator to ensure there was no change in temperature during filtration which could cause 100 some valine to crystallize out. This process was repeated for samples prepared at four different temperatures: $55^{\circ} \mathrm{C}, 40^{\circ} \mathrm{C}$, $30^{\circ} \mathrm{C}$ and $25^{\circ} \mathrm{C}$, giving an indication of the solubility across a wide stretching range of temperatures. Reversed-phase HPLC technique was used for determination of DL-valine concentration. 105 Prepared samples were injected into a Water2697 Separations Module with a 2487 Dual Wavelength detector using Water Symmetry C18 $3.8 \times 150 \mathrm{~mm}$ column. The flow rate within the column was $1 \mathrm{ml} / \mathrm{min}$ and the run time for each sample was 5 minutes. The injection volume was $100 \mu 1$, between each injection 110 the needle was washed with pure deionised water. Each experiment was repeated at least three times and an average solubility was calculated using data from each experiment, as shown in Table 1. 
Table 1 Solubility of DL-valine in water-2-propanol solvent mixture (1:1, $\mathrm{v} / \mathrm{v})$ at different temperatures

\begin{tabular}{ccc}
$\begin{array}{c}\text { Temperature } \\
\left({ }^{\circ} \mathrm{C}\right)\end{array}$ & Solubility $\left(\mathrm{mg} / \mathrm{ml}_{\text {solvent }}\right)$ & Error $(+/-)$ \\
\hline \hline 25 & 14.87 & 0.05 \\
30 & 17.3 & 0.33 \\
40 & 23.5 & 1.02 \\
55 & 38.6 & 1.56
\end{tabular}

Formation of supersaturated solutions

${ }_{5}$ Supersaturated solutions were formed either by rapidly mixing equivolume aliquots of 2-propanol with aqueous valine solution at $25^{\circ} \mathrm{C}$ (antisolvent crystallization) or by cooling a solution prepared at $50^{\circ} \mathrm{C}$ (cooling crystallization).

10 Antisolvent crystallization: A saturated 2-propanol solution was prepared by intense mixing of 2-propanol and excess valine (2-3 g solute in 1-2 1 of solvent) for at least 6 hours in glass bottle. The solutions were filtered by $0.1 \mu \mathrm{m}$ Polytetrafluoroethylene (PTFE) syringe filter using a glass syringe and stored in a glass bottle. 15 Before using these solutions, filtration was applied again to remove any newly formed crystals that may arise from laboratory temperature changes over time.

Aqueous valine solutions were prepared by dissolution of a predetermined weighted amount of valine in distilled water, 20 filtered with a $0.2 \mu \mathrm{m}$ Polypropylene (PP) syringe filter using a glass syringe, and stored in a glass bottle. Fresh aqueous solutions were prepared at least once a week in order to avoid bacterial contamination.

All batch antisolvent crystallization experiments were prepared at ${ }_{25} 25^{\circ} \mathrm{C}$ in small glass vials (diameter $25 \mathrm{~mm}$ ), with volume $5 \mathrm{ml}$ and mixing for 60 , 90 or 180 seconds with magnetic mixer with stirring speed $975 \mathrm{rpm}$, (magnetic stirrer: $12 \mathrm{mmx} 4.5 \mathrm{~mm}$; octagonal). Appropriate volumes of aqueous solution were added to 2-propanol with a pipette; then after mixing, samples were 30 transferred with a pipette into a quartz cuvette for DLS measurements.

Cooling crystallization: A supersaturated solution of DL-valine 70 a)

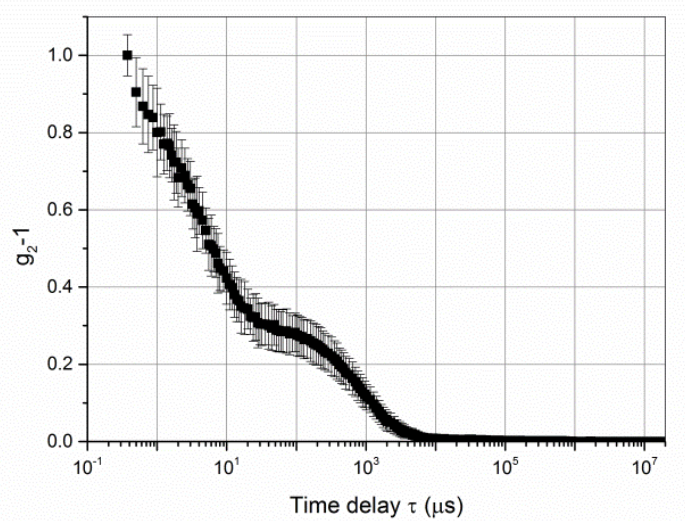

was prepared by dissolution of a predetermined weighed amount 35 of solid valine in distilled water that had been filtered with a $0.2 \mu \mathrm{m}$ Polypropylene (PP) syringe filter using a glass syringe. The aqueous sample was kept in a small glass vial with a screwon cap, at $50^{\circ} \mathrm{C}$ for a few hours. The valine aqueous solution was then mixed with an equal volume of pre-heated 2-propanol that 40 had been filtered with a $0.1 \mu \mathrm{m}$ Polytetrafluoroethylene (PTFE) syringe filter using a glass syringe. Mixtures were typically left for 24 hours at $50^{\circ} \mathrm{C}$ prior to cooling to expel any bubbles. All syringes, filters and cuvettes were preheated in an incubator at $50^{\circ} \mathrm{C}$ to avoid premature cooling of the solution. DLS sample cell 45 holders were preheated to $50^{\circ} \mathrm{C}$ before the solution was inserted. Data collection was started immediately and the temperature ramped down from $50^{\circ} \mathrm{C}$ to $25-15^{\circ} \mathrm{C}$.

\section{Results and discussion}

Supersaturated and undersaturated solutions prepared by cooling ${ }_{50}$ Supersaturated solutions were prepared by mixing hot filtered aqueous valine and 2-propanol at $50^{\circ} \mathrm{C}$, and slowly cooling it to ambient temperature. At $50^{\circ} \mathrm{C}$ only small molecular clusters of up to $1-2 \mathrm{~nm}$ were observed but below about $35^{\circ} \mathrm{C}$ colloidal scale species with mean hydrodynamic diameter of about $200 \mathrm{~nm}$ were 55 found to spontaneously form, accompanied by small molecular clusters (see Figure 1a). The colloidal (mesoscale) clusters formed in cooled solutions had a relatively narrow size distribution (100-300 nm) and on storage over many hours there was no change in their average size or size distribution.

60 After reheating solutions back above $35^{\circ} \mathrm{C}$, mesoscale clusters rapidly disappeared again. As shown in Figure 1b, during both the cooling and heating cycles the sizes of the mesoscale clusters were similar to each other at any particular temperature. Presence of these species cannot be explained by scattering from solid ${ }_{65}$ impurities or bubbles. Heating of a solution typically helps to remove soluble gases, and subsequent cooling would not lead to nucleation of bubbles as the solubility of gases in liquids is expected to increase with decreasing temperature. b)

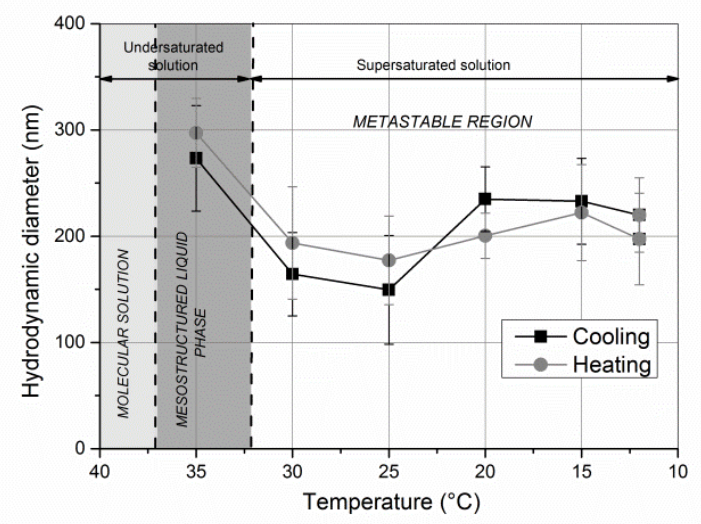

Fig.1 (a) Dynamic light scattering autocorrelation functions $\mathrm{g}_{2}(\tau)-1$ measured at $25^{\circ} \mathrm{C}$, (b) Average hydrodynamic diameter of mesoscale clusters, coolingheating experiment, $\mathrm{C}_{\mathrm{val}}=19 \mathrm{mg} / \mathrm{ml}$, 2-propanol/water, $1: 1$, v/v. 


\section{Cite this: DOI: 10.1039/c0xx00000x}

\section{www.rsc.org/xxxxxx}

Near saturated solutions in contact with solid phase

When valine solutions were cooled to the desired temperature where they were left undisturbed under isothermal quiescent conditions, crystallization was very slow and solutions remained 5 transparent for over many hours until small number of large valine crystals eventually appeared at the bottom of the cell. Even in solutions equilibrated for more than 12 hours, with valine crystals present, the mesoscale clusters persisted. The reversible appearance of the mesoscale clusters in undersaturated solutions 10 at a specific temperature and their coexistence with valine crystals in saturated and supersaturated solutions indicates that an equilibrium phase state has been reached in these solutions.

In another experiment, most of the liquid supernatant in equilibrium with grown crystals was carefully removed with a 15 pipette, without disturbing the crystals, and was replaced with a slightly undersaturated filtered (using $0.1 \mu \mathrm{m}$ PTFE syringe filter)

a)

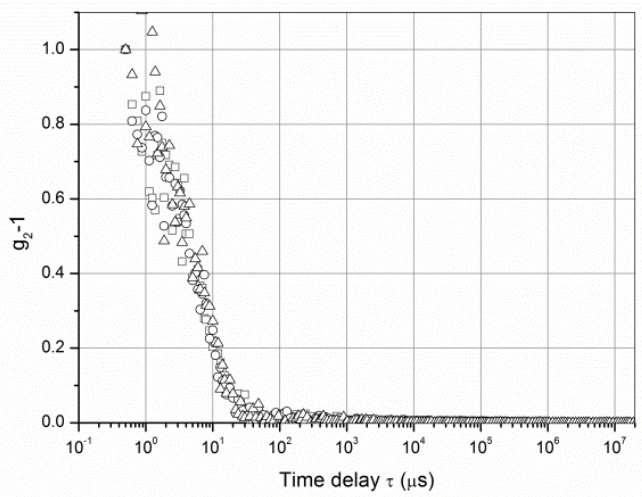

ARTICLE TYPE

valine solution $(14.2 \mathrm{mg} / \mathrm{ml})$. DLS data showed a weak signal from mesoscale clusters still remained so the supernatant was again removed and carefully replaced with the filtered under 20 saturated solution. This treatment lowered the concentration of mesoscale clusters below the detectable limit (Figure 2a).

Crystals were still clearly present bound to the bottom of the cell out of the optical path (the laser beam was about $1 \mathrm{~cm}$ above crystals) and so the system was left undisturbed at $25^{\circ} \mathrm{C}$ for 24 25 hours to equilibrate. After this time DLS data showed a very weak fluctuating signal consistent with the presence of a small population of mesoscale clusters. The undisturbed cell was then gradually heated in the DLS cell whereupon stronger constant signals started to be observed. On reaching $35^{\circ} \mathrm{C}$ the shape of the 30 DLS signal was clearly very similar to that obtained on cooling but still less intense (Figure 2b).

b)

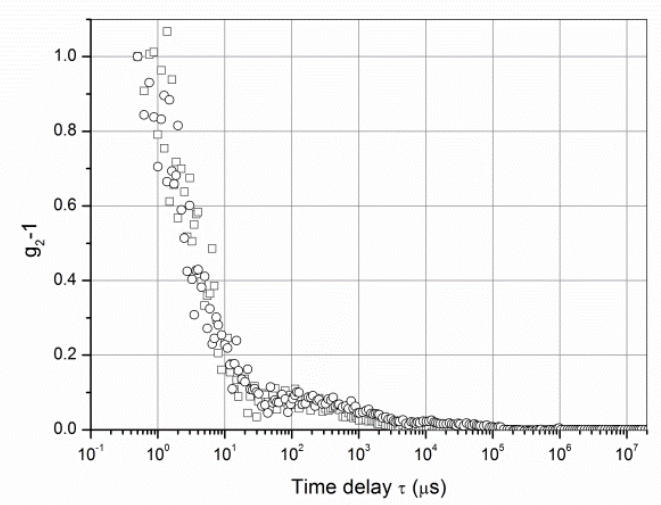

Fig.2 Dynamic light scattering autocorrelation functions $g_{2}(\tau)-1$, a) filtered $(0.1 \mu \mathrm{m}$ PTFE) $14.2 \mathrm{mg} / \mathrm{ml}$ DL-valine/2-propanol/water solution with valine 35 crystals at $25^{\circ} \mathrm{C}$, b) $14.2 \mathrm{mg} / \mathrm{ml}$ DL-valine/2-propanol/water filtered solution with valine crystals at $35^{\circ} \mathrm{C}$, different signs corresponds to repetition of the same experiment.

The appearance of mesoscale clusters on gentle heating is consistent with the source of the mesoscale clusters being the valine crystals at the base of the cell. Diffusion of mesoscale 40 clusters away from the surface of the crystals would be expected to be quite slow in an isothermal stationary cell. However heating provides a convection current that can serve to transport mesoscale clusters into the beam path. Support for this transport limitation was obtained by gentle rocking of the DLS cell to 45 homogenise the mixture without dislodging the crystals. Immediately a much stronger signal was observed for the mesoscale clusters and this could still be observed after a further one week equilibration (Figure 3).

Our observations described above are consistent with formation
50 of mesostructured liquid phases, comprised of mesoscale clusters dispersed within a bulk valine solution. These mesostructured liquid phases appear to be thermodynamically stable over a range of valine concentrations and temperatures. Similar phase behaviour was observed before in aqueous solution of other 55 amino acids, glycine and DL-alanine ${ }^{43}$, and the role of such as structures in the nucleation mechanism of glycine crystals was investigated $^{44}$. The question addressed further here will be, what is the role of the mesoscale clusters in valine crystal nucleation and how the presence of such structure will affect the antisolvent ${ }_{60}$ crystallization process of valine. Before considering that, let us investigate mesoscale clusters in solutions prepared by isothermal mixing of aqueous valine with 2-propanol. 

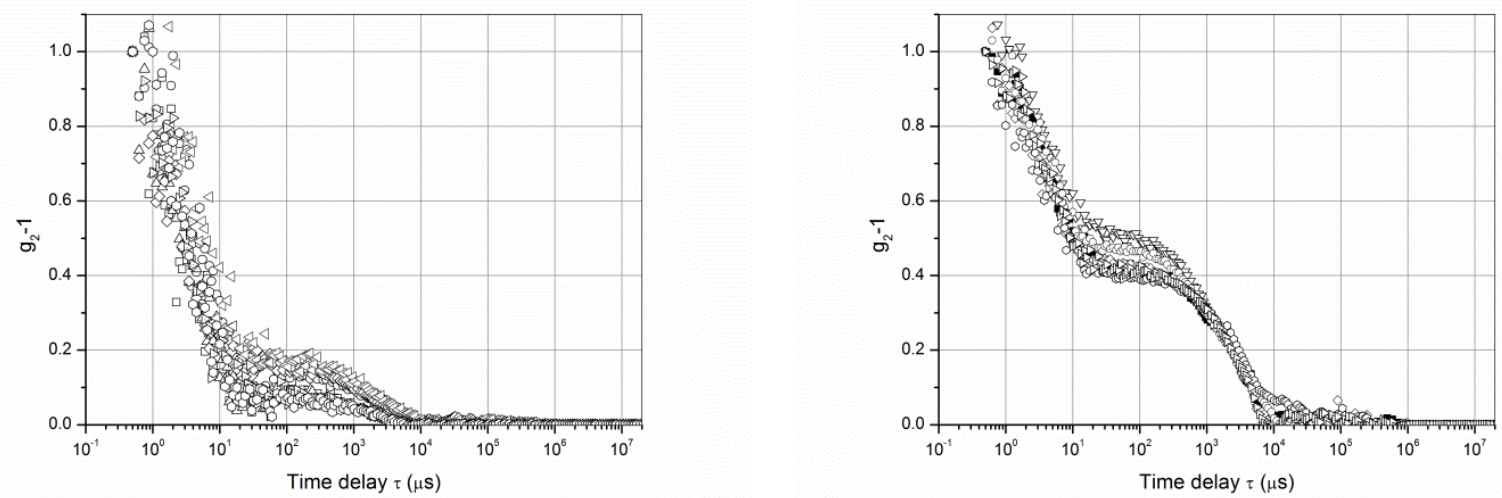

Fig.3 Dynamic light scattering autocorrelation functions $\mathrm{g}_{2}(\tau)-1$, of $14.2 \mathrm{mg} / \mathrm{ml}$ DL-valine/2-propanol/water filtered solution with valine crystals, after 1 week keeping at $25^{\circ} \mathrm{C}$, a) $35^{\circ} \mathrm{C}$, b) $35^{\circ} \mathrm{C}$ after gentle rocking, different signs corresponds to repetition of the same experiment.

\section{${ }_{5}$ Supersaturated and undersaturated solutions prepared by isothermal mixing}

Solutions were prepared by mixing an aqueous valine solution with an equal volume of 2-propanol in a small glass vial, using a magnetic stirring bar, and then transferred to a DLS cell that had 10 been preheated to the same temperature. DLS measurements were then obtained during the induction time period until interference from larger scattering species (e.g., sedimenting crystals) became too intense. Immediately following mixing, small molecular clusters as well as colloidal scale mesoscale clusters could be

15 detected in the optically clear solutions, as in solutions prepared by slow cooling. Control experiments showed that colloidal scale scattering species were not present in filtered solutions prior to mixing. Similarly they could not be detected when mixing aqueous valine and propanol to form dilute solutions at very low 20 supersaturation $(S=0.2)$. The species could not therefore be ascribed to solid impurities or (formation of) (nano)bubbles and were therefore attributable to the presence of valine rich mesoscale clusters. Investigations were carried out on mixtures prepared over range of valine concentrations ranging from 25 undersaturated to supersaturated solutions.

As the valine concentration was increased, the mean size of the mesoscale clusters in supersaturated solutions varied depending upon the method that was used to prepare them. In undersaturated solutions mesoscale clusters were of similar average size 30 regardless of whether they were prepared by cooling or isothermal mixing, although uncertainties in their size estimates were larger because of the fluctuating signals. However, in supersaturated solutions prepared by isothermal mixing the mean cluster size was significantly larger than in those prepared by 35 slow cooling (see Figures 4 and 5). The reasons for the variation of size distribution with the method of solution preparation are not certain but it seems reasonable to assume that larger mesoscale clusters arise from coalescence of smaller ones in metastable supersaturated solutions.

40

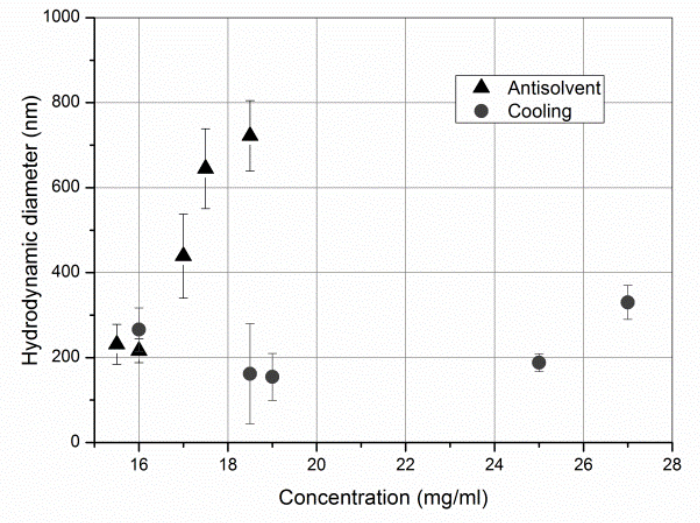

Fig.4 Average hydrodynamic diameter of mesoscale clusters as a function of valine concentration and preparation method: cooling and rapid isothermal mixing (addition to antisolvent), 2-propanol/water, 1:1, v/v.

45

\section{Effect of initial mixing period on induction time}

Solutions were prepared by mixing an aqueous valine solution with an equal volume of 2-propanol in a small glass vial, using a magnetic stirring bar. To determine the mixing efficiency (i.e. 50 how quickly full supersaturation could be achieved on mixing aqueous valine solution with propanol), a competitive reaction procedure was carried out using the same solvent ratios, vials and mixers ${ }^{49}$. These experiments showed that a microscopically homogenous mixture of aqueous solution and propanol was 55 formed in less than 10 seconds. Thus, the observed enhancement of crystallization rates on application of mixing well beyond $10 \mathrm{~s}$ are not attributable to the rate at which supersaturation has been reached.

The agitation was then continued for pre-determined periods to 60 give total agitation times of between 60 and 180 seconds. Typically, $2.5 \mathrm{ml}$ of an aqueous valine solution of known concentration was admixed with $2.5 \mathrm{ml}$ of 2-propanol in a $20 \mathrm{ml}$ vial to give a solution of specified supersaturation. The time before appearance of the first crystals (induction time), was found 65 to vary as a function of the period and method of mixing as well 
as the supersaturation. The induction time was determined by visual inspection based on the appearance of the first crystal in the solution after or during the initial mixing step. Induction times as a function of valine supersaturation for different initial 5 mixing periods are shown in Figure 6. It was observed that the

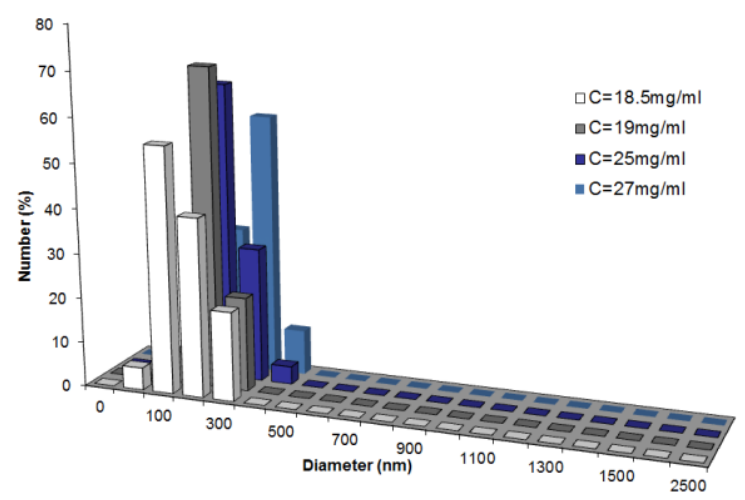

initial mixing period had a large impact on the time to appearance of the first crystals. This effect was retained when the air-solvent interface was eliminated and magnetic stirring was applied within a completely full vial.

Fig.5 Size distribution of mesoscale clusters determined by Brownian microscopy as a function of valine concentration in 2-propanol/water, 1:1, v/v, at $25^{\circ} \mathrm{C}$ : a) solutions prepared by cooling, b) isothermal mixing, mixing time $90 \mathrm{~s}$.

In all these experiments actual formation of the supersaturated 15 solution was very rapid, since it required only seconds to achieve complete mixing of the two fully miscible solvents. Variations in the induction times were therefore influenced by mixing processes carried out over periods 30-50 times greater than this. Such behaviour does not seem to fit with a classical nucleation 20 mechanism because, once a supersaturated solution is produced, the process of molecular association to form critical nuclei is not expected to be affected by the period or intensity of mixing, unless critical nuclei or their precursors are at colloidal length scale. This is because the size of $100 \mathrm{~s} \mathrm{~nm}$ is required for Peclet 25 number, which relates characteristic times of convection and diffusion, to reach an order of unity, and so while molecular clusters on the order of $1 \mathrm{~nm}$ would not be expected to be influenced by shear rates typically encountered in laboratory conditions, mesoscale clusters would be. Furthermore, when the 30 initial mixing conditions were kept identical but the supersaturation varied, a standard plot of log induction time versus log reciprocal of supersaturation squared was not linear, as would be predicted by the classical nucleation theory, see Figure 6.

Molecular crystallization pathways in mesostructured liquid phases

This experimental study has revealed that the three component system of valine dissolved in a 1:1 (v/v) water/2-propanol 40 mixture exhibits a thermodynamically stable mesostructured liquid phase. It should also be noted that the presence of two solvents means that as additional valine is added or as the system is cooled the compositions of mesoscale clusters can adjust in order to minimise the overall free energy of the system. Thus, the 45 distribution of valine, water and 2-propanol between the mesoscale clusters and surrounding solution may be fixed at any particular composition and temperature but continuously variable as the total composition or temperature is changed. This provides a possible reason for why the mesostructured liquid is the most 50 thermodynamically stable phase from the point at which monomers or small molecular cluster assemble to mesoscale clusters (critical mesoscale cluster concentration) ${ }^{43}$ up to the composition where the crystalline phase becomes stable (solubility). At higher concentration the mesostructured liquid 55 phase will continue to be the most kinetically accessible phase but since it is supersaturated it will be metastable with respect to the solid phase.

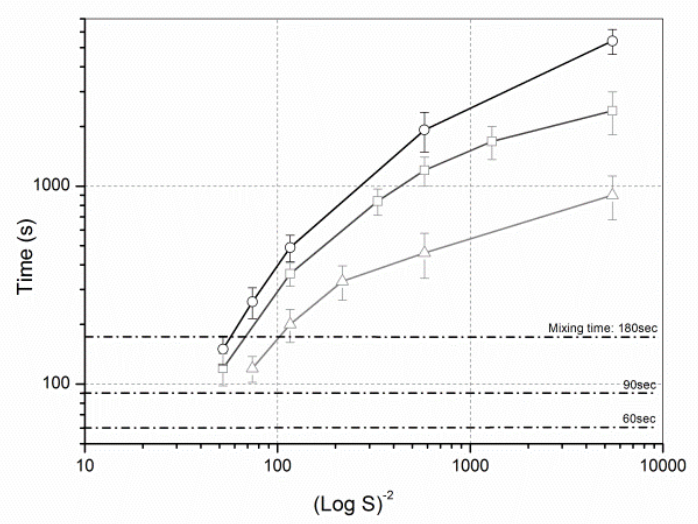

Fig.6 Mean induction times as a function of initial supersaturation, 60 solutions prepared by rapid isothermal mixing, mixing times: $60 \mathrm{~s}$ (circles), $90 \mathrm{~s}$ (squares), $180 \mathrm{~s}$ (triangles), 2-propanol/water, 1:1, v/v. Each experiment was repeated at least 20 times.

In supersaturated solutions prepared by cooling, mesoscale clusters were relatively small and in a narrow size range 100-300 ${ }_{65} \mathrm{~nm}$. Even at relatively high supersaturations all valine solutions prepared by cooling remained optically clear for many hours when left undisturbed. Few large crystals were typically observed after 1-2 days. However, even gentle swirling or pipetting of 
these solutions resulted in rapid onset of nucleation. Although secondary nucleation was considered, it is not expected that gentle flow without any stirrer or impeller would induce massive secondary nucleation in solutions where no visible crystals could 5 be observed. On the other hand, when solutions were prepared by isothermal rapid initial mixing followed by quiescent isothermal crystallization, induction times were at least two orders of magnitude shorter than in solutions of the identical composition prepared by cooling. We have also seen that application of rapid 10 initial mixing results in much larger size of mesoscale clusters (Figures 4 and 5).

We hypothesize that once mesoscale clusters reach a critical size, a much more rapid nucleation process can be accessed and crystallization follows quickly. Thus the greater the number of 15 large mesoscale clusters formed the faster the overall rate of crystal formation will be. It can be speculated that large mesoscale clusters may form by shear induced coalescence during the intial mixing period. Alternatively, adsorption of mesoscale clusters onto the vial surface may well provide an 20 alternate albeit slower coalescence pathway. Surface assisted coalescence would then trigger crystal nucleation in a similar way. Consistent with this hypothesis it was often observed that valine crystals formed from cooled solutions were bound to the side of the vial. Figure 7 provides a pictorial summary of the 25 proposed nucleation crystallization pathways.

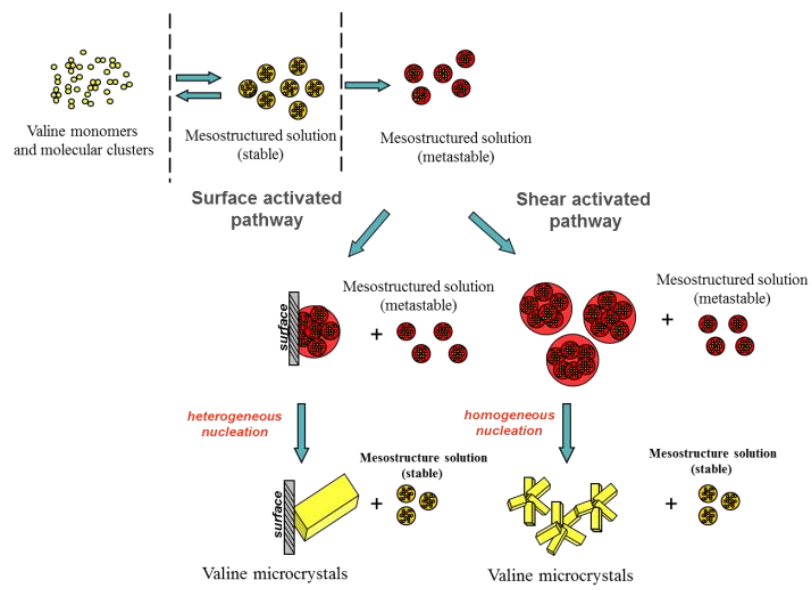

Fig.7 Proposed pathways for crystallization of amino acid (DL-valine) involving mesoscale clusters: surface and shear activated pathways.

Borrowing from the classical nucleation theory and building on 30 what we proposed previously ${ }^{44}$ smaller mesoscale clusters may not contain sufficient amount of valine to be able to form stable nuclei able to survive and grow in the surrounding bulk solution. This could certainly be the case if as anticipated the mesoscale clusters were to contain a significant volume fraction of solvent. 35 The critical mesoscale cluster size for productive nucleation would then correspond to the cluster volume that contains just sufficient amount of valine to be able to form a viable growing crystal. In this model it can be imagined that mesoscale clusters of all sizes intermittently sample the crystalline phase and most 40 commonly they simply reform as the nascent crystal is not viable in the bulk solution. Thus the probability of a nucleation event occurring within small supersaturated mesoscale clusters, such as formed on cooling, will be very low. Only when a critical cluster volume has been reached will the probability of productive
45 nucleation become high enough for it to occur at a significant rate.

\section{Conclusions}

In this work we have experimentally studied antisolvent and cooling crystallization of valine from water/2-propanol mixtures. ${ }_{50}$ DLS data showed that valine solutions contain two kinds of clusters present in the optically clear solution: molecular clusters (diameter of up to 1-2nm) and solute-rich mesoscale clusters (diameter of hundreds nanometers). Within cooled solutions mesoscale clusters were around $200 \mathrm{~nm}$ in diameter and remained 55 unchanged in size and monodisperse over periods of many hours. Samples prepared by isothermal rapid initial mixing contained larger mesoscale clusters and a significant effect of the initial mixing step used to form supersaturated valine solutions on the mesoscale cluster size was observed. Solutions prepared by rapid 60 isothermal mixing crystallized at least two orders of magnitude faster than solution of identical compositions prepared by cooling. Careful experimentation showed that mesoscale clusters dispersed within bulk valine solution together form a thermodynamically stable mesostructured liquid phase that at the 65 crystal solubility limit exists in equilibrium with valine crystals.

That the mesostructured liquid is a genuine phase of matter can be demonstrated by the appearance of stable mesoscale clusters on mixing filtered solvent with an excess of valine crystals. The mesoscale clusters were found to arise directly from dissolution 70 of solid showing that the solid and mesostructured liquid phases are present together in thermodynamic equilibrium. Although they are clearly visible using DLS it is important to recognise that total amount of valine contained within the mesoscale clusters is very small and careful experimental design was required to obtain

75 these data. Large mesoscale clusters obtained by shear or surface assisted coalescence are proposed to be the likely location of productive nucleation events leading to formation of valine crystals.

\section{Notes and references}

${ }_{80}{ }^{a}$ EPSRC Centre for Innovative Manufacturing in Continuous Manufacturing and Crystallization, Department of Chemical and Process Engineering, University of Strathclyde, 75 Montrose Street, Glasgow G1 IXJ, UK. Tel: +44 (0)141 548 2410;

E-mail: anna.jawor-baczynska@strath.ac.uk, jan.sefcik@strath.ac.uk $85{ }^{b}$ WestCHEM, Department of Pure and Applied Chemistry, University of Strathclyde, 295 Cathedral Street, Glasgow G1 1XL, UK. Tel: +44 (0)141 548 2301;

E-mail:b.d.moore@strath.ac.uk

90 1. R. J. Davey, W. Liu, M. J. Quayle and G. J. T. Tiddy, Crystal Growth \& Design, 2002, 2, 269-272.

2. S. Chattopadhyay, D. Erdemir, J. M. B. Evans, J. Ilavsky, H. Amenitsch, C. U. Segre and A. S. Myerson, Crystal Growth \& Design, 2005, 5, 523-527.

95 3. D. Erdemir, S. Chattopadhyay, L. Guo, J. Ilavsky, H. Amenitsch, C. U. Segre and A. S. Myerson, Physical Review Letters, 2007, 99, 115702.

4. S. Parveen, R. J. Davey, G. Dent and R. G. Pritchard, Chemical Communications, 2005, 1531-1533. 
5. R. A. Chiarella, A. L. Gillon, R. C. Burton, R. J. Davey, G. Sadiq, A. Auffret, M. Cioffi and C. A. Hunter, Faraday Discussions, 2007, 136.

6. T. J. Sorensen, P. C. Sontum, J. Samseth, G. Thorsen and D. Malthe-

5 Sorenssen, Chemical Engineering\&Technology, 2003, 26, 307-312.

7. R. C. Burton, E. S. Ferrari, R. J. Davey, J. Hopwood, M. J. Quayle, J. L. Finney and D. T. Bowron, Crystal Growth \& Design, 2008, 8, 1559-1565.

10 8. S. Hamad, C. E. Hughes, C. R. A. Catlow and K. D. M. Harris, Journal of Physical Chemistry B, 2008, 112, 7280-7288.

9. R. J. Davey, K. Allen, N. Blagden, W. I. Cross, H. F. Lieberman, M. J. Quayle, R. Righini, L. Seton and G. J. T. Tiddy, Crystal Engineering Communications, 2002, 257-264.

15 10. J. F. Lutsko and G. Nicolis, Physical Review Letters, 2006, 96, 046102/046101-046102/046104.

11. P. G. Vekilov, Interdisciplinary Transport Phenomena, 2009, 1161, 377-386.

12. J. Sefcik and A. V. McCormick, Chemical Engineering Science, $20 \quad$ 1999, 54, 3513-3519.

13. A. S. Myerson and P. Y. Lo, Journal of Crystal Growth, 1990, 99, 1048-1052.

14. J. W. Mullin and C. L. Leci, Philosophical Magazine, 1969, 19, 1075-1077.

25 15. S. Parveen, R. J. Davey, G. Dent and R. G. Pritchard, Chemical Communications, 2005, 1531-1533.

16. R. J. Davey, G. Dent, R. K. Mughal and S. Parveen, Crystal Growth \& Design, 2006, 6, 1788-1796.

17. R. A. Chiarella, A. L. Gillon, R. C. Burton, R. J. Davey, G. Sadiq, A.

30 Auffret, M. Cioffi and C. A. Hunter, Faraday Discussions, 2007, 136, 179-193.

18. S. Hamad, C. E. Hughes, C. R. A. Catlow and K. D. M. Harris, The Journal of Physical Chemistry B, 2008, 112, 7280-7288.

19. R. J. Davey, S. L. M. Schroeder and J. H. ter Horst, Angewandte

35 Chemie International Edition, 2013, n/a-n/a.

20. D. Gebauer, M. Kellermeier, J. D. Gale, L. Bergstrom and H. Colfen, Chemical Society Reviews, 2014.

21. P. G. Vekilov, Crystal Growth \& Design, 2004, 4, 671-685.

22. D. Erdemir, A. Y. Lee and A. S. Myerson, Accounts of Chemical $40 \quad$ Research, 2009, 42, 621-629.

23. G. Gliko, W. Pan, P. Katsonic, N. Neumaier, O. Galein, S. Weinkauf and P. G. Vekilov, Journal of Physical Chemistry B, 2007, 11, 3106-3114.

24. M. B. Sweatman, R. Fartaria and L. Lue, The Journal of Chemical $45 \quad$ Physics, 2014, 140, 124508.

25. C. Forsyth, P. A. Mulheran, C. Forsyth, M. D. Haw, I. S. Burns and J. Sefcik, Crystal Growth \& Design, 2015, 15. 94-102.

26. D. Erdemir, A. Y. Lee and A. S. Myerson, Accounts of Chemical Research, 2009, 42, 621-629.

50 27. P. E. Bonnett, K. J. Carpenter, S. Dawson and R. J. Davey, Chemical Communications, 2003, 6, 698-699.

28. P. Rein den Wolde and D. Frenkel, Science, 1997, 277, 1975-1978.

29. V. G. Taratuta, A. Holschbach, G. M. Thurston, D. Blankschtein and G. B. Benedek, Journal of Physical Chemistry 1990, 94,

55 2140-2144.
30. D. F. Rosenbaum and C. F. Zukoski, Journal of Crystal Growth, 1996, 169, 752-758.

31. N. Asherie, N. Lomakin and G. B. Benedek, Physical Review Letters, 1996, 77, 4832-4835.

60 32. H. L. Chen, J. C. Hwang, J. M. Yang and R. C. Wang, Polymer, 1998, 39, 6983-6989.

33. Y. S. Soh, J. H. Kim and C. C. Gryte, Polymer, 1995, 36, 3711-3717.

34. X. Wang, J. M. Gillian and D. J. Kirwan, Crystal Growth \& Design, 2006, 6, 2214-2227.

65 35. X. Wang and D. J. Kirwan, Crystal Growth \& Design, 2006, 6, $2228-$ 2240.

36. J. Liu and Å. C. Rasmuson, Crystal Growth \& Design, 2013, 13, 4385-4394.

37. A. W. Xu, Y. Ma and H. Cölfen, Journal of Materials Chemistry, 2007, 17, 415-449.

38. M. Niederberger and H. Cölfen, Physical Chemistry Chemical Physics, 2006, 8, 3271-3287.

39. H. Cölfen and S. Mann, Angewandte Chemie, 2003, 42, 2350-2365.

40. D. D. Medina and Y. Mastai, Crystal Growth \& Design, 2008, 8, 75 3646-3651.

41. D. Schwahn, Y. Ma and H. Cölfen, Journal of Physical Chemistry Letters C, 2007, 111, 3224-3227.

42. Y. Ma, H. Cölfen and M. Antonietti, Journal of Physical Chemistry $B, 2006,110,10822-10828$.

80 43. A. Jawor-Baczynska, B. D. Moore, H. S. Lee, A. V. McCormick and J. Sefcik, Faraday Discussions, 2013, 167, 425-440.

44. A. Jawor-Baczynska, J. Sefcik and B. D. Moore, Crystal Growth \& Design, 2013, 13, 470-478.

45. M. Variny, S. Alvarez de Miguel, B. D. Moore and J. Sefcik, Journal 85 of Dispersion Science and Technology, 2008, 29, 617-620.

46. P. Linder, T. Zember and P. N. Pusey, Neutron, X-rays and light scattering methods to soft condensed matter. Introduction to Scattering experiments, Elsevier Science B.V., 2002.

47. R. Finsy, Advances in Colloid and Interface Science, 1994, 52, 7990143.

48. R. Finsy, L. Deriemaeker, E. Geladé and J. Joosten, Journal of Colloid and Interface Science, 1992, 153, 337-354.

49. A. Brown, PhD Thesis, University of Strathclyde, 2012. 